\title{
MAXIMUM LIKELIHOOD BINARY DETECTION IN IMPROPER COMPLEX GAUSSIAN NOISE
}

\author{
Amirhossein S.Aghaei, Konstantinos N.Plataniotis, Subbarayan Pasupathy \\ E-mail: \{aghaei, kostas, pas\} @ comm.utoronto.ca \\ The Edward S. Rogers Sr. Dept. of Electrical and Computer Engineering \\ University of Toronto
}

\begin{abstract}
In a wide range of communication systems, including DS-CDMA and OFDM systems, the signal-of-interest might be corrupted by an improper [1] (also called non circularly symmetric [2]) interfering signal. This paper studies the maximum likelihood (ML) detection of binary signals in the presence of additive improper complex Gaussian noise. Proposing a new measure for noncircularity of complex random variables, we will derive the ML decision rule and its performance based on this measure. It will be shown that the ML detector performs pseudo correlation [1] as well as conventional correlation of the observation to the signals-of-interest. As an alternative solution, we will propose a filter for converting improper signals to proper ones, called circularization filter, and will utilize it together with a conventional matched-filter (MF) to construct an ML detector.
\end{abstract}

Index Terms - Maximum likelihood detection, improper complex, Gaussian noise, circularization, matched filters

\section{INTRODUCTION}

Since the first studies of improper complex signals (also called non circularly symmetric signals) by [1,2] there has been a growing interest on characterization of such signals in various applications, especially communication systems. The issue of improper interference was firstly studied by [3] in the context of multi-access interference (MAI) in synchronous DS-CDMA systems using BPSK modulation. However, improper interferences are not restricted to this case and can arise in many other applications (e.g. $[4,5,6])$.

This paper considers the simple scalar case of binary transmission over a typical additive Gaussian noise channel, where the noise is independent of the transmitted signal. Let's assume that $r$ is a scalar $^{1}$ noisy observation of the unknown scalar signal-of-interest $s$ which might take either of the deterministic constant values $s_{0}$ or $s_{1}$ with equal probabilities according to the following hypotheses:

$$
\left\{\begin{array}{lll}
H_{0} & : & r=s_{0}+n \\
H_{1} & : & r=s_{1}+n
\end{array}\right.
$$

where $r, s_{0}, s_{1}, n \in \mathbb{C}$ and the noise $n$ has the following variance and pseudo-variance [1]:

$$
\sigma_{n}^{2} \triangleq \mathrm{E}\left\{|r-\bar{r}|^{2}\right\} \quad, \quad \gamma_{n}^{2} \triangleq \mathrm{E}\left\{(r-\bar{r})^{2}\right\} .
$$

\footnotetext{
${ }^{1}$ In this paper we use the following notation: lowercase letters for scalar variables (e.g. $z$ ), boldface lowercase letters for vectors (e.g. z), and boldface uppercase letters for matrices (e.g. Z). $\mathbb{R}$ and $\mathbb{C}$ represent the real and complex domains. $\Re\{z\}$ and $\Im\{z\}$ represent the real and imaginary parts of a complex variable $z . \sigma_{z}^{2}, \gamma_{z}^{2}$, and $\sigma_{x y}$ represent the variance of $z$, pseudo variance of $z$, and the covariance between $x$ and $y$, respectively.
}

The pseudo variance $\gamma_{n}^{2}$ is in fact the covariance between $n$ and $n^{*}$. When $n$ is uncorrelated from $n^{*}$ (i.e. $\gamma_{n}^{2}=0$ ), it will be called a proper or circularly symmetric noise. This paper focuses on the problem of detecting the transmitted signal, which is deterministic but unknown at the receiver, from the noisy observation $r$ when the noise $n$ is improper. Although [7] has studied the mean square estimation and detection of improper signals-of-interest observed in proper noise, the problem of ML detection in presence of improper noise has not been studied in general yet, except some works which have studied the special case of DS-CDMA systems with BPSK signaling (e.g. $[4,8]$ ). However, the results of this paper are applicable to any binary signaling with fixed constellation in the complex plain (e.g. antipodal or orthogonal signaling).

In Section 3, after derivation of ML decision rule, we will prove that the ML detector makes use of the correlation existing between real and imaginary parts of the interfering noise to get the optimal detection performance. Based on the results of Section 3, we will propose a circularization filter for converting the improper additive noise into a proper one in Section 4. This filter can be used as a preprocessing step for conventional tools (MFs in this paper) to enable them to deal with improper noises.

\section{IMPROPER GAUSSIAN RANDOM VARIABLES}

Let $z$ be a scalar complex Gaussian random variable (RV) with Cartesian representation of the form $z=x+j y$, where $x$ and $y$ are two real valued RVs. It has been shown in [9] that $z$ can be completely characterized by either $\mathbf{w}_{z} \triangleq[x, y]^{T}$ or $\mathbf{v}_{z} \triangleq \frac{1}{\sqrt{2}}\left[z, z^{*}\right]^{T}$, as these two random vectors relate to each other through a unitary linear transformation:

$$
\mathbf{w}_{z}=\mathbf{T v}_{z}, \quad \text { where } \quad \mathbf{T}=\frac{1}{\sqrt{2}}\left[\begin{array}{cc}
1 & 1 \\
-j & j
\end{array}\right]
$$

Moreover, complete second-order characterization of $z$ requires knowledge of the following statistics [9]:

$$
\sigma_{z}^{2}=\sigma_{x}^{2}+\sigma_{y}^{2} \quad, \quad \gamma_{z}^{2}=\left(\sigma_{x}^{2}-\sigma_{y}^{2}\right)+j\left(2 \sigma_{x y}\right),
$$

or knowledge of the following covariance matrices:

$$
\begin{aligned}
& \mathbf{C}_{\mathbf{v}_{z} \mathbf{v}_{z}^{H}} \triangleq \mathrm{E}\left\{\left(\mathbf{v}_{z}-\mathbf{v}_{\bar{z}}\right)\left(\mathbf{v}_{z}-\mathbf{v}_{\bar{z}}\right)^{H}\right\}=\frac{1}{2}\left[\begin{array}{cc}
\sigma_{z}^{2} & \gamma_{z}^{2} \\
\left(\gamma_{z}^{2}\right)^{*} & \sigma_{z}^{2}
\end{array}\right] \\
& \mathbf{C}_{\mathbf{w}_{z} \mathbf{w}_{z}^{T}} \triangleq \mathrm{E}\left\{\left(\mathbf{w}_{z}-\mathbf{w}_{\bar{z}}\right)\left(\mathbf{w}_{z}-\mathbf{w}_{\bar{z}}\right)^{T}\right\}=\left[\begin{array}{cc}
\sigma_{x}^{2} & \sigma_{x y} \\
\sigma_{x y} & \sigma_{y}^{2}
\end{array}\right] .
\end{aligned}
$$

Note that by knowing one of the covariance matrices in (5) or (6), the other one can be determined since $\mathbf{C}_{\mathbf{w}_{z} \mathbf{w}_{z}^{T}}=\mathbf{T C}_{\mathbf{v}_{z} \mathbf{v}_{z}^{H}} \mathbf{T}^{H}$. 
Finally, it should be noted that the probability density function (pdf) of a complex Gaussian RV $z$ is defined in [9] as follows:

$$
\begin{aligned}
p_{\mathbf{z}}(z) & =\left|2 \pi \mathbf{C}_{\mathbf{w}_{z} \mathbf{w}_{z}^{T}}\right|^{-\frac{1}{2}} \exp \left\{-\frac{1}{2}\left\|\mathbf{w}_{z}-\mathbf{w}_{\bar{z}}\right\|_{\mathbf{C}_{\mathbf{w}_{z} \mathbf{w}_{z}^{T}}^{-1}}\right\} \\
& =\left|2 \pi \mathbf{C}_{\mathbf{v}_{z} \mathbf{v}_{z}^{H}}\right|^{-\frac{1}{2}} \exp \left\{-\frac{1}{2}\left\|\mathbf{v}_{z}-\mathbf{v}_{\bar{z}}\right\|_{\mathbf{C}_{\mathbf{v}_{z} \mathbf{v}_{z}^{-1}}^{-1}}\right\},
\end{aligned}
$$

where $\|\mathbf{x}\|_{\mathbf{C}} \triangleq \mathbf{x}^{H} \mathbf{C x}$.

The latter equation in (4) reveals that $z$ is proper $\left(\gamma_{z}^{2}=0\right)$ if the power of $z$ is equally distributed between its real and imaginary parts $\left(\sigma_{x}^{2}=\sigma_{y}^{2}\right)$ and there exists no correlation between these two parts $\left(\sigma_{x y}=0\right)$. In this case, we will get $\gamma_{z}^{2}=0$ and $\sigma_{z}^{2}=2 \sigma_{x}^{2}$, and the covariance matrices of (5) and (6) will be reduced to: $\mathbf{C}_{\mathbf{w}_{z} \mathbf{w}_{z}^{T}}=$ $\mathbf{C}_{\mathbf{v}_{z} \mathbf{v}_{z}^{H}}=\left(\sigma_{z}^{2} / 2\right) \mathbf{I}$, where $\mathbf{I}$ is the identity matrix. In the following definition, we will propose a new complex valued measure for noncircularity (or improperness) of RVs, which will be utilized in analyzing the ML detector in next sections.

Definition 1 Given a complex $R V z$, the noncircularity coefficient of $z$, denoted by $\alpha_{z}$, is defined as the ratio of its pseudo-variance to its variance:

$$
\alpha_{z} \triangleq \frac{\gamma_{z}^{2}}{\sigma_{z}^{2}}=\left(\frac{\sigma_{x}^{2}-\sigma_{y}^{2}}{\sigma_{x}^{2}+\sigma_{y}^{2}}\right)+j\left(\frac{2 \sigma_{x y}}{\sigma_{x}^{2}+\sigma_{y}^{2}}\right) .
$$

We call $\alpha_{z}$ the noncircularity coefficient of $z$, in that its real and imaginary parts are normalized measures of the power difference between $x$ and $y$ and the correlation between $x$ and $y$, respectively. Therefore, $\alpha_{z}$ is independent from the changes in the power of $z$ and only conveys information about its properness. In fact, decomposition of the pseudo-variance as $\gamma_{z}^{2}=\sigma_{z}^{2} \alpha_{z}$ provides us with the ability to distinguish between changes in $\gamma_{z}^{2}$ caused by changing the power of $z$ as opposed to changes caused by changing the properness of $z$.

Theorem 1 The magnitude of the pseudo-variance of a complex $R V$ is upper bounded by the value of its conventional variance.

(Proof: Appendix-A) Theorem-1 implies that the newly defined coefficient $\alpha_{z}$ lies within the unit circle (i.e. $0 \leq\left|\alpha_{z}\right| \leq 1$ ), and the extreme case for improperness of $z$ occurs when $\left|\gamma_{z}^{2}\right|=\sigma_{z}^{2}$.

\section{MAXIMUM LIKELIHOOD BINARY DETECTION IN THE PRESENCE OF IMPROPER NOISE}

In this paper we consider the binary hypothesis model of (1) and assume that the detector has complete knowledge of the noise statistics (i.e. $\bar{n}$ as well as either $\mathbf{C}_{\mathbf{v}_{n} \mathbf{v}_{n}^{H}}$ or $\mathbf{C}_{\mathbf{w}_{n} \mathbf{w}_{n}^{T}}$ ); hence, the noise pdf $p_{N}(n)$ is available. Without loss of generality, we will assume that $n$ has zero mean (For nonzero mean noises, we can use $r^{\prime}=r-\bar{n}$ ). Under the maximum likelihood paradigm, the following decision rule could be defined:

$$
L(r)=\frac{p_{R \mid S_{1}}\left(r \mid s_{1}\right)}{p_{R \mid S_{0}}\left(r \mid s_{0}\right)} \underset{\hat{s}=s_{0}}{\stackrel{\hat{s}=s_{1}}{\gtrless}} 1,
$$

By conditioning on $s_{j}$, the detector assumes that $s_{j}$ is transmitted; therefore, $p_{R \mid S_{j}}\left(r \mid s_{j}\right)=p_{N}\left(r-s_{j}\right)$ since $s_{j}$ is a deterministic constant value. By substituting

$$
p_{R \mid S_{j}}\left(r \mid s_{j}\right)=\left|2 \pi \mathbf{C}_{\mathbf{v}_{n} \mathbf{v}_{n}^{H}}\right|^{-\frac{1}{2}} \exp \left\{-\frac{1}{2}\left\|\mathbf{v}_{r}-\mathbf{v}_{s_{j}}\right\|_{\mathbf{C}_{\mathbf{v}_{n} \mathbf{v}_{n}^{H}}^{-1}}\right\}
$$

in (9) and taking the natural logarithm of both sides, the following decision rule will be resulted:

$$
\begin{aligned}
& \mathbf{v}_{s_{1}}^{H} \mathbf{C}_{\mathbf{v}_{n} \mathbf{v}_{n}^{H}}^{-1} \mathbf{v}_{r}-\frac{1}{2} \mathbf{v}_{s_{1}}^{H} \mathbf{C}_{\mathbf{v}_{n} \mathbf{v}_{n}^{H}}^{-1} \mathbf{v}_{s_{1}} \\
& \stackrel{\hat{s}=s_{1}}{\gtrless} \mathbf{v}_{s_{0}}^{H} \mathbf{C}_{\mathbf{v}_{n} \mathbf{v}_{n}^{H}}^{-1} \mathbf{v}_{r}-\frac{1}{2} \mathbf{v}_{s_{0}}^{H} \mathbf{C}_{\mathbf{v}_{n} \mathbf{v}_{n}^{H}}^{-1} \mathbf{v}_{s_{0}},
\end{aligned}
$$

where $\mathbf{v}_{s_{j}}=\frac{1}{\sqrt{2}}\left[s_{j}, s_{j}^{*}\right]^{T}, \mathbf{v}_{n}=\frac{1}{\sqrt{2}}\left[n, n^{*}\right]^{T}, \mathbf{v}_{r}=\frac{1}{\sqrt{2}}\left[r, r^{*}\right]^{T}$, and

$$
\mathbf{C}_{\mathbf{v}_{n} \mathbf{v}_{n}^{H}}^{-1}=\frac{2}{\sigma_{z}^{2}\left(1-\left|\alpha_{n}\right|^{2}\right)}\left[\begin{array}{cc}
1 & -\alpha_{n} \\
-\alpha_{n}^{*} & 1
\end{array}\right] .
$$

By substituting these values in (10), we will get

$$
\begin{aligned}
& \Re\left\{\left(r s_{1}^{*}-\frac{1}{2}\left|s_{1}\right|^{2}\right)-\left(r s_{1}-\frac{1}{2} s_{1}^{2}\right) \alpha_{n}^{*}\right\} \\
& \underset{\hat{s}=s_{0}}{\gtrless} \Re\left\{\left(r s_{0}^{*}-\frac{1}{2}\left|s_{0}\right|^{2}\right)-\left(r s_{0}-\frac{1}{2} s_{0}^{2}\right) \alpha_{n}^{*}\right\} \text {. }
\end{aligned}
$$

Fig. 1(a) shows the the block diagram of this detector. The term $r s_{j}^{*}$ correlates the observation $r$ with $s_{j}$, while the the term $r s_{j}$ pseudo correlates [1] $r$ with $s_{j}$. After the outputs of the correlator and pseudo correlator are adjusted by $\frac{1}{2}\left|s_{j}\right|^{2}$ and $\frac{1}{2} s_{j}^{2}$, respectively, they will be superposed according to the noncircularity coefficient $\alpha_{n}$, and the ML decision will be based on the real part of this result. For proper noises, the outputs of pseudo correlators for both $s_{1}$ and $s_{0}$ are ignored (multiplied by $\alpha_{n}=0$ ), and the decision only depends on the outputs of correlators according to

$$
\Re\left\{\left(r s_{1}^{*}-\frac{1}{2}\left|s_{1}\right|^{2}\right)\right\} \underset{\hat{s}=s_{0}}{\stackrel{\hat{s}=s_{1}}{\gtrless}} \Re\left\{\left(r s_{0}^{*}-\frac{1}{2}\left|s_{0}\right|^{2}\right)\right\}
$$

which is the decision rule of well-known conventional ML detectors for proper noises.

By using (3), decision rule of (10) can also be rewritten as:

$$
\begin{aligned}
\mathbf{w}_{s_{1}}^{T} \mathbf{C}_{\mathbf{w}_{n} \mathbf{w}_{n}^{T}}^{-1} \mathbf{w}_{r}-\frac{1}{2} \mathbf{w}_{s_{1}}^{T} \mathbf{C}_{\mathbf{w}_{n} \mathbf{w}_{n}^{T}}^{-1} \mathbf{w}_{s_{1}} & \\
& \stackrel{\substack{s=s_{1} \\
\hat{s}=s_{0}}}{\gtrless} \mathbf{w}_{s_{0}}^{T} \mathbf{C}_{\mathbf{w}_{n} \mathbf{w}_{n}^{T}}^{-1} \mathbf{w}_{r}-\frac{1}{2} \mathbf{w}_{s_{0}}^{T} \mathbf{C}_{\mathbf{w}_{n} \mathbf{w}_{n}^{T}}^{-1} \mathbf{w}_{s_{0}},
\end{aligned}
$$

where $\mathbf{w}_{s_{j}}=\left[\Re\left\{s_{j}\right\}, \Im\left\{s_{j}\right\}\right]^{T}, \mathbf{w}_{n}=[\Re\{n\}, \Im\{n\}]^{T}, \mathbf{w}_{r}=$ $[\Re\{r\}, \Im\{r\}]^{T}$, and

$$
\mathbf{C}_{\mathbf{w}_{n} \mathbf{w}_{n}^{T}}^{-1}=\frac{2}{\sigma_{n}^{2}\left(1-\left|\alpha_{n}\right|^{2}\right)}\left[\begin{array}{cc}
1-\Re\left\{\alpha_{n}\right\} & -\Im\left\{\alpha_{n}\right\} \\
-\Im\left\{\alpha_{n}\right\} & 1+\Re\left\{\alpha_{n}\right\}
\end{array}\right] .
$$

The ML decision rule of (13) can be interpreted as follows. $\Re\{r\}$ and $\Im\{r\}$ can be considered as two observations of signals $\Re\left\{s_{j}\right\}$ and $\Im\left\{s_{j}\right\}$ which are corrupted with noises $\Re\{n\}$ and $\Im\{n\}$, respectively. When $n$ is improper $\left(\alpha_{n} \neq 0\right)$, the off-diagonal elements of $\mathbf{C}_{\mathbf{w}_{n} \mathbf{w}_{n}^{T}}$ are not zero and $\Re\{n\}$ and $\Im\{n\}$ will become correlated noises.

Therefore, the term $\mathbf{w}_{s_{1}}^{T} \mathbf{C}_{\mathbf{w}_{n} \mathbf{w}_{n}^{T}}^{-1} \mathbf{w}_{r}\left(=\mathbf{v}_{s_{j}}^{H} \mathbf{C}_{\mathbf{v}_{n} \mathbf{v}_{n}^{H}}^{-1} \mathbf{v}_{r}\right)$ can be viewed as a whitened matched-filter for optimal joint detection of $\Re\left\{s_{j}\right\}$ and $\Im\left\{s_{j}\right\}$ from the noisy observation vector of $\mathbf{w}_{r}$, when the elements of the jointly Gaussian noise vector $\mathbf{w}_{n}$ are correlated to each other. This relationship between ML detector for improper scalar noise $n$ and the well-known whitened MF for correlated $\mathbf{w}_{n}$ motivates us to study another alternative implementation of optimal ML detector in the next section. 


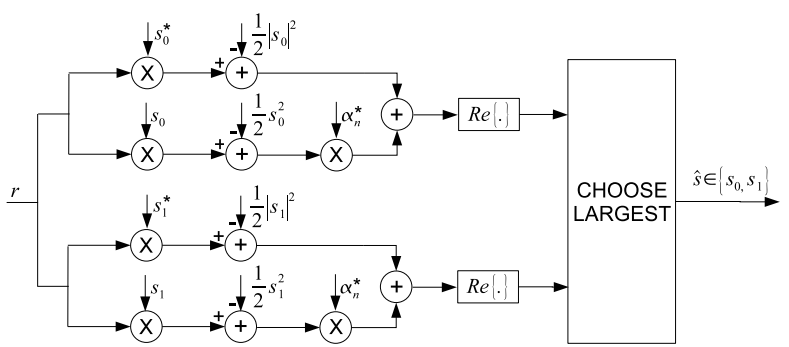

(a) Combination of Correlators and Pseudo Correlators

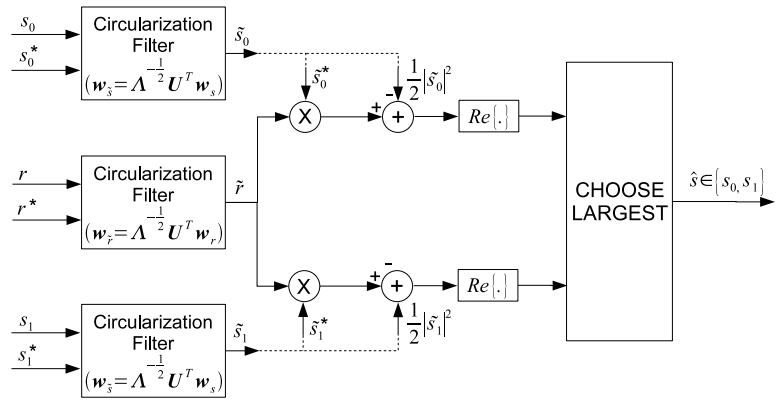

(b) Circularization Filter followed by Matched-Filter

Fig. 1. Equivalent implementations of binary ML detector.

Finally, it should be mentioned that for proper noises, decision rules of (10) and (13) reduce to

and

$$
\mathbf{v}_{s_{1}}^{H} \mathbf{v}_{r}-\frac{1}{2} \mathbf{v}_{s_{1}}^{H} \mathbf{v}_{s_{1}} \underset{\hat{s}=s_{0}}{\stackrel{\hat{s}=s_{1}}{\gtrless}} \mathbf{v}_{s_{0}}^{H} \mathbf{v}_{r}-\frac{1}{2} \mathbf{v}_{s_{0}}^{H} \mathbf{v}_{s_{0}}
$$

$$
\mathbf{w}_{s_{1}}^{T} \mathbf{w}_{r}-\frac{1}{2} \mathbf{w}_{s_{1}}^{T} \mathbf{w}_{s_{1}} \underset{\hat{s}=s_{0}}{\stackrel{\hat{s}=s_{1}}{\gtrless}} \mathbf{w}_{s_{0}}^{T} \mathbf{w}_{r}-\frac{1}{2} \mathbf{w}_{s_{0}}^{T} \mathbf{w}_{s_{0}},
$$

both of which make use of conventional MFs $\left(\mathbf{v}_{s_{j}}^{H} \mathbf{v}_{r}\right.$ or $\left.\mathbf{w}_{s_{j}}^{T} \mathbf{w}_{r}\right)$.

\section{CIRCULARIZATION FILTER FOLLOWED BY MATCHED-FILTER}

In Section 3, it was shown that an improper scalar noise $n$ can be represented by the noise vector $\mathbf{w}_{n}$ with non i.i.d. elements. Consequently, by converting the elements of $\mathbf{w}_{n}$ into i.i.d elements, the corresponding noise will become proper. Thus, we can exploit the concept of whitening filters in order to convert an improper noise into a proper one as follows.

Definition 2 Given an improper complex $R V z$, the circularization (or proprization) filter is defined as the filter which converts $z$ into a proper $R V \widetilde{z}$, using the following input/output relationship:

$$
\mathbf{w}_{\widetilde{z}}=\boldsymbol{\Lambda}^{-\frac{1}{2}} \mathbf{U}^{T} \mathbf{w}_{z},
$$

where $\mathbf{U}$ and $\mathbf{\Lambda}$ are the matrices including eigenvectors and eigenvalues of $\mathbf{C}_{\mathbf{w}_{z} \mathbf{w}_{z}^{T}}$, respectively (i.e. $\mathbf{C}_{\mathbf{w}_{z} \mathbf{w}_{z}^{T}}=\mathbf{U} \boldsymbol{\Lambda} \mathbf{U}^{T}$ ).

According to Appendix-B, this filter firstly rotates the coordinate system and uses the eigenvectors of $\mathbf{C}_{\mathbf{w}_{z} \mathbf{w}_{z}^{T}}$ as the new coordinate system to remove the correlation between $\Re\{z\}$ and $\Im\{z\}$. Afterwards, the new coordinates should be scaled separately by the inverse of the square root of corresponding eigenvalues to get a proper $\widetilde{z}$ with equal power on $\Re\{\widetilde{z}\}$ and $\Im\{\widetilde{z}\}$.

Applying a circularization filter to the observation $r$, we get

$$
\mathbf{w}_{\widetilde{r}}=\Lambda^{-\frac{1}{2}} \mathbf{U}^{T} \mathbf{w}_{r}=\boldsymbol{\Lambda}^{-\frac{1}{2}} \mathbf{U}^{T} \mathbf{w}_{s}+\boldsymbol{\Lambda}^{-\frac{1}{2}} \mathbf{U}^{T} \mathbf{w}_{n}=\mathbf{w}_{\widetilde{s}}+\mathbf{w}_{\widetilde{n}},
$$

where $\widetilde{s}$ and $\widetilde{n}$ denote the transformed signal and the circularized noise $\left(\mathbf{C}_{\mathbf{w}_{\tilde{n}} \mathbf{w}_{\tilde{n}}^{T}}=\mathbf{I}\right)$, and $\mathbf{U}$ and $\boldsymbol{\Lambda}$ are derived from eigen decomposition of $\mathbf{C}_{\mathbf{w}_{n} \mathbf{w}_{n}^{T}}$. As it is shown in Fig. 1(b), the ML detection of $\widetilde{s}$ can now be accomplished using conventional matching of $\widetilde{r}$ to $\widetilde{s}^{*}$, i.e.,

$$
\Re\left\{\widetilde{r} \widetilde{s}_{1}^{*}-\frac{1}{2}\left|\widetilde{s}_{1}\right|^{2}\right\} \underset{\substack{\hat{s}=s_{1} \\ \gtrless}}{\gtrless}\left\{\left\{\widetilde{r} \widetilde{s}_{0}^{*}-\frac{1}{2}\left|\widetilde{s}_{0}\right|^{2}\right\}\right.
$$

or equivalently

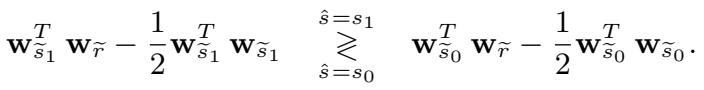

Note that both decision rules of (16) and (17) are equivalent to the ML decision rule of (10), in that

$$
\Re\left\{\widetilde{r} \widetilde{s}_{j}^{*}\right\}=\mathbf{w}_{\widetilde{s}_{j}}^{T} \mathbf{w}_{\widetilde{r}}=\mathbf{w}_{s_{j}}^{T} \mathbf{C}_{\mathbf{w}_{n} \mathbf{w}_{n}^{T}}^{-1} \mathbf{w}_{r}=\mathbf{v}_{s_{j}}^{H} \mathbf{C}_{\mathbf{v}_{n} \mathbf{v}_{n}^{H}}^{-1} \mathbf{v}_{r}
$$

Accordingly, we will call the term $\mathbf{v}_{s_{j}}^{H} \mathbf{C}_{\mathbf{v}_{n} \mathbf{v}_{n}^{H}}^{-1} \mathbf{v}_{r}$ a circularized MF to distinguish it from conventional $\mathrm{MF}\left(\mathbf{v}_{s_{j}}^{H} \mathbf{v}_{r}\right)$.

In fact, circularization is a reversible preprocessing step and preserves all the data included in $\mathbf{v}_{r}$. Thus, applying the ML criterion to the preprocessed data will yield the same result as applying the ML criterion to the original data (see [10] Page 289).

Finally, it should be noted that for proper noises $\left(\alpha_{n}=0\right)$, we have $\mathbf{C}_{\mathbf{w}_{\tilde{n}} \mathbf{w}_{\tilde{n}}^{T}}=\left(\sigma_{n}^{2} / 2\right) \mathbf{I}$. Consequently, the circularization filter simply scales $r$ with factor $\sqrt{2 / \sigma_{n}^{2}}$. Therefore, for proper noises, it can be omitted and the optimal detector will be the conventional MF.

\section{PERFORMANCE}

Let $r^{\prime}=x^{\prime}+j y^{\prime}$ be the representation of the observation $r=$ $x+j y$ in a new coordinate system which has the following axes: the line passing through the points $s_{0}$ and $s_{1}$, and the perpendicular bisector of the line segment $s_{0} s_{1}$. Assuming that the angle between the former line and $\mathrm{x}$-axis is $\theta$, we have $\left[x^{\prime}, y^{\prime}\right]^{T}=\mathbf{R}_{w}(-\theta)[x-$ $\Re\{\bar{s}\}, y-\Im\{\bar{s}\}]^{T}$, where $\mathbf{R}_{w}(-\theta)$ is defined in Appendix-B and $\bar{s}=\frac{1}{2}\left(s_{0}+s_{1}\right)$. It can be shown that when $\mathrm{x}^{\prime}$-axes is parallel to one of the eigenvectors of $\mathbf{C}_{\mathbf{w}_{n} \mathbf{w}_{n}^{T}}$, the ML detectors of Sections 3,4 will become equivalent to conventional MF. For all other cases, however, the ML detector is not a conventional MF and outperforms it. It can be proved that the probability of detection error is

$$
\begin{aligned}
P_{e} & =Q\left(\sqrt{\frac{2\left|s_{1}-s_{0}\right|^{2}}{\sigma_{r}^{2}} \times \frac{1-\left|\alpha_{r}\right| \cos (\varphi-2 \theta)}{1-\left|\alpha_{r}\right|^{2}}}\right) \\
& =Q\left(\sqrt{\frac{\left|s_{1}-s_{0}\right|^{2}}{4 \sigma_{x^{\prime}}^{2}\left(1-\rho^{\prime 2}\right)}}\right),
\end{aligned}
$$

where $\rho^{\prime}=\sigma_{x^{\prime} y^{\prime}} / \sqrt{\sigma_{x^{\prime}}^{2} \sigma_{y^{\prime}}^{2}}$ and $\varphi$ are the correlation factor of the noise in the $\mathrm{x}^{\prime}-\mathrm{y}^{\prime}$ coordinates and the phase of the noncircularity coefficient, respectively. Equation (18) together with Appendix-B 


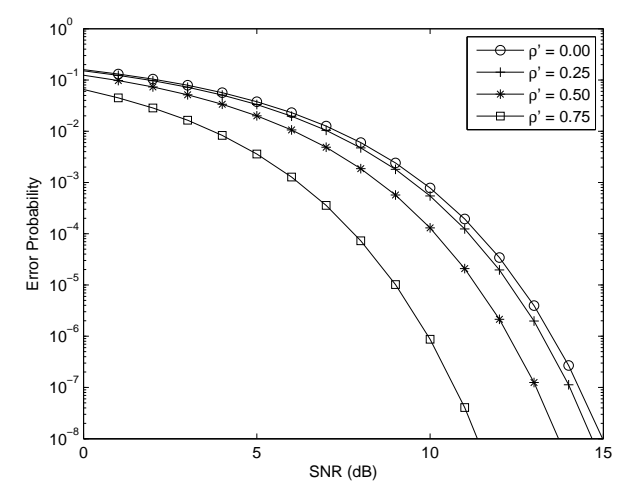

Fig. 2. Error Probability for different values of $\rho^{\prime}$ when $\sigma_{x^{\prime}}^{2}=\sigma_{y^{\prime}}^{2}$ and $s_{1}=-s_{0} \in \mathbb{R}$.

reveals that for a fixed power constraint, the optimal signaling is antipodal signaling $\left(s_{1}=-s_{0}\right)$ along the eigenvector corresponding to the smallest eigenvalue of $\mathbf{C}_{\mathbf{w}_{n} \mathbf{w}_{n}^{T}}$. As mentioned before, the ML detector for this signaling will become a conventional MF.

In order to illustrate how ML detectors of Section 3 outperform conventional MFs, we will consider an antipodal signaling such that $\theta \neq(\varphi-\pi) / 2$ and $\theta \neq \varphi / 2$ (in these two cases the ML detector is MF). For simplicity, let's assume that $\theta=0$ and the power of noise is equally distributed in $\mathrm{x}^{\prime}$ and $\mathrm{y}^{\prime}$ directions. Fig. 2 presents the performance of the ML detector in this case for different values of $\rho^{\prime}$. It can be seen that for improper noises with higher $\rho^{\prime}$, this performance improves since the optimal detector is provided with more side information in $y^{\prime}$.

Fig. 2 also illustrates the improvement which can be achieved by using ML detectors instead of conventional MFs. In $x^{\prime}-y^{\prime}$ coordinate system, the imaginary part of $s^{\prime}$ is zero for both $s_{1}$ and $s_{0}$. Therefore, a conventional MF, which is based on a proper noise model, assumes that there is no relevant data in the imaginary part of the observation $\left(y^{\prime}\right)$; hence, ignores it. The performance of such detector is well-known as $Q\left(\sqrt{\left|s_{1}-s_{0}\right|^{2} / 4 \sigma_{x^{\prime}}^{2}}\right)$, which does not change for different values of $\rho^{\prime}$ and is always equivalent to the case of $\rho^{\prime}=0$ shown in Figure 2. However, using the correct improper model for the noise, the ML detector takes advantage of the correlation existing between the real and imaginary parts of observation $r^{\prime}$ to get optimal performance. Thus, its performance improves for improper noises when $\rho^{\prime}$ increases.

\section{CONCLUSION AND REMARKS}

In this paper, the ML detection of binary signals in additive improper Gaussian noise was studied. Using the noncircularity coefficient of the noise, the ML detector combines correlation and pseudo correlation of $r$ with $s$ during the detection process. As a more general solution, we proposed that instead of designing a new ML detector for improper noises, we can equip conventional detectors, which are designed for proper noises, with a circularization filter. Finally, it was shown that the resulting ML detector in both approaches become a conventional MF when the noise is proper or the signaling is along eigenvectors of $\mathbf{C}_{\mathbf{w}_{n} \mathbf{w}_{n}^{T}}$.

Due to space limitations and for simplicity, this paper was restricted to scalar RVs and binary hypotheses; however, the more general results for random vectors and $M$-ary hypotheses as well as derivation of error probability will be presented in future works.

\section{A. PROOF OF THEOREM-1}

According to (4) we have $\sigma_{z}^{4}-\left|\gamma_{z}^{4}\right|=4\left[\sigma_{x}^{2} \sigma_{y}^{2}-\sigma_{x y}^{2}\right]$. From Schwartz inequality $\left(\sigma_{x}^{2} \sigma_{y}^{2}-\sigma_{x y}^{2} \geq 0\right)$, it follows that $0 \leq\left|\gamma_{z}^{4}\right| \leq$ $\sigma_{z}^{4}$; hence, $0 \leq\left|\gamma_{z}^{2}\right| \leq \sigma_{z}^{2}$.

\section{B. EIGEN DECOMPOSITION OF $\mathbf{C}_{\mathbf{v}_{Z} \mathbf{v}_{Z}^{H}} A N D \mathbf{C}_{\mathbf{w}_{Z} \mathbf{w}_{Z}^{T}}$}

Let $\mathbf{C}_{\mathbf{v}_{z} \mathbf{v}_{z}^{H}}=\mathbf{Q} \boldsymbol{\Lambda}_{1} \mathbf{Q}^{H}$ and $\mathbf{C}_{\mathbf{w}_{z} \mathbf{w}_{z}^{T}}=\mathbf{U} \boldsymbol{\Lambda}_{2} \mathbf{U}^{T}$ be the eigen decomposition of covariance matrices defined in (6), (5), where $\boldsymbol{\Lambda}_{1}$ and $\boldsymbol{\Lambda}_{2}$ are diagonal matrices including the eigen values of $\mathbf{C}_{\mathbf{v}_{z} \mathbf{v}_{z}^{H}}$ and $\mathbf{C}_{\mathbf{w}_{z} \mathbf{w}_{z}^{T}}$ in non-increasing order, and $\mathbf{Q}$ and $\mathbf{U}$ are unitary matrices including their corresponding eigenvectors. Then

$$
\begin{aligned}
& \text { 1. } \boldsymbol{\Lambda}_{1}=\boldsymbol{\Lambda}_{2}=\boldsymbol{\Lambda}=\frac{\sigma_{z}^{2}}{2}\left[\begin{array}{cc}
1+\left|\alpha_{z}\right| & 0 \\
0 & 1-\left|\alpha_{z}\right|
\end{array}\right] \\
& \text { 2. } \mathbf{U}=\left[\begin{array}{cc}
\cos \frac{\varphi}{2} & -\sin \frac{\varphi}{2} \\
\sin \frac{\varphi}{2} & \cos \frac{\varphi}{2}
\end{array}\right]=\mathbf{R}_{w}\left(\frac{\varphi}{2}\right) \\
& \text { 3. } \mathbf{Q}=\mathbf{T}^{H} \mathbf{U}=\left[\begin{array}{cc}
e^{j \frac{\varphi}{2}} & 0 \\
0 & e^{-j \frac{\varphi}{2}}
\end{array}\right] \mathbf{T}^{H}=\mathbf{R}_{v}\left(\frac{\varphi}{2}\right) \mathbf{T}^{H}
\end{aligned}
$$

where $\varphi$ denotes the phase of noncircularity coefficient $\alpha_{z}$, and $\mathbf{R}_{w}\left(\frac{\varphi}{2}\right)$ and $\mathbf{R}_{v}\left(\frac{\varphi}{2}\right)$ are unitary transformations on $\mathbf{w}_{z}$ and $\mathbf{v}_{z}$, respectively, both of which result in the rotation of $z$ by phase $\frac{\varphi}{2}$.

\section{REFERENCES}

[1] F. D. Neeser and J. L. Massey, "Proper complex random processes with applications to information theory," IEEE Trans. Inf. Theory, vol. 39, pp. 1293-1302, July 1993.

[2] B. Picinbono, "On circularity," IEEE Trans. Signal Process., vol. 42, pp. 3473-3482, Dec. 1994.

[3] Y. C. Yoon and H. Leib, "Maximizing SNR in improper complex noise and applications to CDMA," IEEE Commun. Lett., vol. 1, pp. 5-8, Jan. 1997.

[4] G. E. Bottomley, "CDMA downlink interference suppression using I/Q projection," IEEE Trans. Wireless Commun., vol. 2, pp. 890-900, Sept. 2003.

[5] G. Taubock, "Noise analysis of DMT," in Global Telecom. Conf., 2003. IEEE, vol. 4, pp. 2136-2140, Dec. 2003.

[6] R. Nilsson, F. Sjoberg, and J. P. LeBlanc, "A rank-reduced LMMSE canceller for narrowband interference suppression in OFDM-based systems," IEEE Trans. Commun., vol. 51, pp. 2126-2140, Dec. 2003.

[7] P. J. Schreier, L. L. Scharf, and C. T. Mullis, "Detection and estimation of improper complex random signals," IEEE Trans. Inf. Theory, vol. 51, pp. 306-312, Jan. 2005.

[8] Y. Yoon and H.-M. Kim, "Maximum likelihood multiuser detection of DS/CDMA signals in improper noise," in 2005 Fifth Int. Conference on Inf., Commun. and Signal Process., pp. 224-228, Dec. 2005.

[9] B. Picinbono, "Second-order complex random vectors and normal distributions," IEEE Trans. Signal Process., vol. 44, pp. 2637-2640, Oct. 1996.

[10] H. L. Van Trees, Detection, Estimation, and Modulation Theory, vol. I: Detection, Estimation, and Linear Modulation Theory. John Wiley and Sons, 1968. 